\title{
Laws of Large Numbers for Dynamical Systems with Randomly Matched Individuals
}

\author{
RICHARD T. BOYLAN* \\ Washington University, Olin School of Business, \\ St. Louis, Missouri 63130
}

Received November 26, 1990; revised September 26, 1991

\begin{abstract}
Biologists and economists have analyzed populations where each individual interacts with randomly selected individuals. The random matching generates a very complicated stochastic system. Consequently biologists and economists have approximated such a system with a deterministic system. The justification for such an approximation is that the population is assumed to be very large and thus some law of large numbers must hold. This paper gives a characterization of random matching schemes for countably infinite populations. In particular this paper shows that there exists a random matching scheme such that the stochastic system and the deterministic system are the same. Journal of Economic Literature Classification Numbers: 026, 213. (C) 1992 Academic Press, lnc.
\end{abstract}

\section{INTRODUCTION}

There is a large literature in economics, evolutionary biology, and population genetics that studies dynamical systems with individuals randomly matched in pairs, although the particular way in which people are matched is left unspecified. In this paper we describe ways of matching individuals and the properties of such matching schemes. We first give some examples in economics and biology where random matching occurs.

Gale [13] considers a market that contains a large number of individuals who meet randomly in pairs. Agents are characterized by their history, which includes the initial endowments and utility function. When individuals meet, they bargain over the terms of trade. Gale assumes that the matching scheme is such that the probability with which an individual meets an individual with a particular history equals the proportion of the

* This paper could not have been written without the help of Roko Aliprantis and especially Kim Border. I also thank for their help: Mahmoud El-Gamal, Richard McKelvey, John Nachbar, Stanley Sawyer, Thomas Wolff, the associate editor, and the referee. Financial support was provided by the John Randolph Haynes and Dora Haynes Fellowship and the Alfred P. Sloan Dissertation Fellowship and is duly appreciated. This paper is based on Chapter 2 of my dissertation. 
population with that particular history. Furthermore, Gale assumes that the distribution of histories does not depend on which individuals were matched in the previous periods. Gale goes on to characterize the equilibrium strategies.

Fudenberg and Levine [10] examine a model where there are $n$ populations; each population consists of $m$ different types, where each type is defined by a belief over which strategy the other individuals adopt. The proportion of population $i$ that is of type $j$ is denoted by $p_{i j}$. Every period each player from a population $i$ is randomly and independently matched with one individual from every other population $i^{\prime}\left(i^{\prime} \neq i\right)$. Fudenberg and Levine assume that the probability with which a player meets a player from population $i^{\prime}$ and of type $j$ is $p_{i^{\prime} j}$. The randomly matched individuals play a game selecting strategies according to their beliefs and updating their beliefs according to the observed strategy choices of the other players. Fudenberg and Levine go on to characterize the steady states of this dynamic system.

These models are very similar to the models that have been studied extensively in population genetics. ${ }^{1}$ In a common formulation of these models, the phenotype (e.g., eye color) is determined by the action of two genes at one locus. Genes are assumed to be of two types (alleles): $A_{1}$ and $A_{2}$. Individuals are of three types (genotypes): $A_{1} A_{1}, A_{1} A_{2}$, and $A_{2} A_{2}$. When two individuals mate they each produce gametes (reproductive cells). Gametes receive one of the parent's genes. An offspring is produced by the union of a gamete from each parent. When two individuals of types $a a^{\prime}$ and $b b^{\prime}$ mate they produce offspring of type $a b, a b^{\prime}, a^{\prime} b$, and $a^{\prime} b^{\prime}$ with equal probability. Another assumption describes which individuals mate. "Matings take place at random with respect to the genetic differences being considered and in a population of infinite size" [4, p. 45]. All individuals mate at the same time and then are completely replaced by their offspring. Thus the dynamics of the process depends on the random matching of individuals and the random selection of genotypes for the offspring. Suppose there is a large population and the initial relative frequency of alleles $A_{1}$ and $A_{2}$ in the population is $p_{1}$ and $p_{2}\left(p_{1}+p_{2}=1\right)$. Then the Hardy-Weinberg theorem states that in the next period the relative frequency of the genotypes $A_{1} A_{1}, A_{1} A_{2}, A_{2} A_{2}$ is respectively $p_{1}^{2}, 2 p_{1} p_{2}$, and $p_{2}^{2}$. This theorem is "proved" (just as in the original papers by Hardy [16] and Weinberg [31]) by computing the expected proportion with which each of the matches occurs. No explicit modeling of the matching scheme and no derivation of a law of large numbers is given. Furthermore there seems to be some confusion in the literature about whether the

\footnotetext{
${ }^{1}$ This similarity is onc of the rcasons for the large literature on the application of evolutionary biology to game theory. See $[1,2,5,6,9,11,22,28]$.
} 
population needs to be infinite or very large. Feller [8, p. 135] writes: "In a large population the actually observed frequencies of the three genotypes in the filial generation will be close to the theoretical probabilities." And in a footnote at the end of this sentence: "The statement is made precise by the law of large numbers and the central limit theorem, which permits us to estimate the effect of chance fluctuations." Hofbauer and Sigmund [17] write: "A few more premises were used implicitly in the derivation. For instance we equated "frequency" with "probability." This is admissible in the limiting case of very large populations" [page 9]. The model just described is generalized to the case where genotypes differ in fitness (the expected number of offspring) and thus where the number of a particular allele changes with time.

Thus, underlying the models by Fudenberg and Levine as well as the biological models is the conjecture that if the population is very large (possibly infinite) there exists a matching scheme such that the proportion of matches involving individuals of two particular types is equal to the expected number of such matches when all matches are equally likely. In the model by Fudenberg and Levine and in the biological models, the matching scheme is implemented repeatedly and each individual's type is allowed to change between periods. Then the individuals follow a stochastic process governed by the way types are updated and by the matching rule. Again, the models are analyzed as deterministic systems. The law of motion is computed by assuming that in every period the set of individuals adopting the same strategy is matched with the population average. Thus there is an implicit conjecture that if the population is large enough, there exists a matching rule that matches individuals in every period in such a way that the deterministic process provides a good description of the stochastic process.

This paper proves that there is a random matching rule such that the deterministic process is equivalent to the stochastic process when individuals are represented by the set of natural numbers. Thus this paper answers the following questions that are usually left unanswered in the literature: How is the population characterized? What is the structure of the matching process? How are types assigned to individuals? What do we mean by each subpopulation facing the distribution of types equal to the population distribution? How do wc characterize the evolution of the population from the random matching scheme?

The problem analyzed in this paper is similar to the problem of whether idiosyncratic risk disappears in large populations. If the population is taken to be the natural numbers and individuals' risk are i.i.d., then the population as a whole does not face idiosyncratic risk. Feldman and Gilles [7] and Judd [18] point out that this result does not necessarily extend to the case where the population is taken to be the unit interval. Let $X_{i}$ be the risk 
faced by individual $i$ and let $X=\left(X_{i}\right)_{i \in[0,1]}$ be a collection of i.i.d. random variables that have finite mean and finite variance. Then it can be shown $[19$, p. 10$]$ that there does not exist a measurable process equivalent to $X$, and thus the integral of $X$ does not converge (almost surely) to its expectation. $^{2}$

The discussion of the risk problems leads us to think that analyzing random matching in a countable population is easier than analyzing random matching when the population is the unit interval. Furthermore, in the models of risk analyzed, the event that individual $i$ is in an accident does not affect the probability that individual $j$ is in an accident, while in matching models, if individual $i$ is matched to individual $j$, then individual $j$ has to be matched to individual $i$. Thus, the problem of randomly matching individuals seems harder than the problem of randomly assigning accidents to individuals. Consequently, it seems more likely that useful results can be generated in the countable population model than in the continuum population model.

Section 2 gives an example of how the dynamics for a very large population differs from the dynamics for an infinite population. Section 3 describes the problem of finding a matching technology for infinite populations such that all matches are equally likely. Section 4 describes the main results of this paper. Section 5 proves the first conjecture; i.e., that there is a matching scheme such that a law of large numbers holds. Sections 6, 7, 8.3 prove the second conjecture; i.e., that there exists a random matching scheme such that the law of large numbers holds when individuals are matched infinitely many times. Section 8.2 proves that the deterministic process provides a good approximation for the evolution of a finite (but large enough) population, for a finite number of periods.

\section{Matches Over Very Large Populations}

A natural argument for supporting the conjecture in the introduction is the following: if the population is of size $n$ then the probability that the matching rule does not behave as its expectation is $q(n)$. By the law of large numbers $q(n)$ can be made arbitrarily small by taking $n$ large. In other words for large populations the conjecture is approximately correct. As we will see in Section 8.2 this argument can be formalized if the matching scheme occurs finitely many times. In many cases analyzed in the literature, however, the matching scheme analyzed occurs infinitely many times, and it is thus possible that the small perturbations that occur in each period

\footnotetext{
${ }^{2}$ However we can get $L_{2}$-convergence (see Uhlig [29]). Another way around the problem is to index individuals by elements of an abstract nonatomic probability space (see Green $[15]$ ).
} 
alter the process significantly in the limit. We construct an example where this problem actually occurs. The example is taken from evolutionary biology because (i) such models are very important in evolutionary biology, (ii) several economists have applied evolutionary models to game theory, and (iii) the mathematics in this example are very tractable.

Suppose that there is a population consisting of $3 M$ individuals, where $M$ is an even number. Individuals have a very simple life: an individual born at time $t$ interacts with one randomly selected individual, then at time $t+1$ gives birth to new individuals and dies. The matching scheme is left unspecified but we assume that the same random matching scheme is used in each period and that in each period all matches can occur with positive probability. ${ }^{3}$ Individuals are of three different types: $s_{1}, s_{2}, s_{3}$. If an individual of type $s_{r}$ interacts with an individual of type $s_{v}$ then the $s_{r}$-individual has $a_{r v}$ offspring while the $s_{v}$-individual has $a_{\mathrm{v} r}$ offspring. All offspring are of the same type as the parent. Suppose that the matrix $A=\left(a_{v w}\right)$ is as follows:

$$
A=\left(\begin{array}{lll}
1 & 2 & 0 \\
0 & 1 & 2 \\
2 & 0 & 1
\end{array}\right) .
$$

First note that the population size stays constant: if individuals of type $s_{v}$ and $s_{w}$ meet they will have together $2=a_{v w}+a_{w v}$ offspring and thus keep the population size constant. Note also that, because of the 0 entry in the matrix $A$, at any period $t$, there is a positive probability that one of the types disappears, which we denote by $q_{e}^{\prime}$. For any population distribution among types $\left(M_{1}, M_{2}, M_{3}\right)$, where $M_{1}+M_{2}+M_{3}=3 M$, there exists a set of matches for which one of the types totally disappears. Since all matches are possible these matches will have positive probability. Denote the probability that one type disappears when the population distribution is $\left(M_{1}, M_{2}, M_{3}\right)$ by $q_{M_{1}, M_{2}, M_{3}}$. Let

$$
q_{e}=\min _{\left\{M_{1}+M_{2}+M_{3}=3 M\right\}} q_{M_{1}, M_{2}, M_{3}} .
$$

Then, for all $t, q_{e}^{t} \geqslant q_{e}>0$.

Note that if a type disappears, it never comes back. Consequently, if the matching scheme is repeated infinitely often, the probability that at least one of the three types will disappear is at least

$$
1-\lim _{t \rightarrow \infty}\left(1-q_{e}\right)^{t}=1 \text {. }
$$

\footnotetext{
${ }^{3}$ One way of describing the random matching scheme is to think of individuals as being drawn successively (without replacement) from an urn. The first and second individuals drawn are matched together, the third and fourth individuals drawn are matched together, and so on.
} 
Let $B_{t}$ be the set of events for which one of the types disappears by time $t$. From the previous remarks $\left\{B_{t}\right\}$ is an increasing sequence and $\lim _{t \rightarrow \infty} P\left(B_{t}\right)=1$; therefore one of the types disappears in finite time. It is easy to see that if type $s_{v}$ disappears, then the population will converge to a population composed uniquely of individuals of type $s_{v+1(\bmod 3)}$ (almost surely). Therefore the population will converge to one of the vertices of the simplex in finite time (almost surely). Since this result is true irrespective of the population size $3 M$, it will also be true as $M$ tends to infinity.

In evolutionary biology the evolution for the population is approximated by the replicator model. The replicator model assumes that a proportion $2 p_{v} p_{w}$ of the matches are between individuals of type $s_{v}$ and $s_{w}$, where $p_{v}$ is the proportion of the population of type $s_{v}$. Then the proportion of the population of type $i$ at time $t+1$ is related to the population at time $t$ in the following way:

$$
p_{r}^{t+1}=R_{r}\left(p^{t}\right) \equiv p_{r}^{t} \frac{\sum_{v} p_{v}^{t} a_{r v}}{\sum_{v} \sum_{w} p_{v}^{t} a_{v w} p_{w}^{t}} .
$$

Biologists are interested in the behavior of the system as times goes to infinity (see [21]). We already know the limit behavior of the stochastic process for the matrix considered above. Next we characterize the dynamics of the replicator model for the same matrix. We are particularly interested in whether the replicator model gives a good approximation of the evolution of a large population. First suppose that the initial population is composed of a third of each type. Then the population will remain at the barycenter, contrary to the behavior of the stochastic process. The next proposition (which is proven in the Appendix) characterizes the limiting population when the system does not start at the barycenter.

Proposition 1. Suppose the initial population is in the interior of the simplex and is not at the barycenter. Then the set of accumulation points of the trajectory is a subset of the boundary which contains the vertices and infinitely many points.

Thus for any period $T$, there is a period $t>T$ such that $p^{t}$ is far removed from any of the vertices, and we conclude that the deterministic approximation does not give a very good approximation of the stochastic process.

In most of the literature, the evolution of the population is studied through a continuous approximation of (1). Since

$$
p_{r}^{t+1}-p_{r}^{t}=p_{r}^{t} \frac{\sum_{v} p_{v}^{t} a_{r v}-\sum_{v} \sum_{w} p_{v}^{t} a_{v w} p_{w}^{t}}{\sum_{v} \sum_{w} p_{v}^{t} a_{v w} p_{w}^{t}},
$$


it is claimed that the discrete process can be replaced by the differential equation,

$$
\frac{d p_{r}}{d t}=p_{r}\left(\sum_{r} p_{v} a_{r v}-\sum_{v} \sum_{w} p_{v} a_{v w} p_{w}\right)
$$

(see for instance [30]). For the matrix considered above, it can be shown (see [32]) that the trajectories of the continuous approximation are simple closed curves going through the initial population. The behavior of the continuous approximation is thus different from the stochastic process and the discrete deterministic process.

\section{Matches Over Countably InTinite Number or Agents}

This section introduces the notation that will be used in this paper and discusses the problems of finding a random matching scheme for an infinite population. We assume that the population is countably infinite and is denoted by

$$
N=\{1,2, \ldots, n, \ldots\} .
$$

Each individual is matched anonymously to exactly one other individual. There are $m$ types of individuals. We keep the terminology vague so that the discussion applies to economics (where types are beliefs, preferences, or endowments), evolutionary biology (where types are strategies), and to population genetics (where types are genotypes or alleles). The set of types is denoted by $S$, where

$$
S=\left\{s_{1}, \ldots, s_{m}\right\} .
$$

For convenience we represent the set of types by the standard basis for $\mathbf{R}^{m}$; i.e., $s_{r}$ is the $m$ dimensional vector with a one on the $r$ th component and zeros on the other components. Let $\alpha: \mathbf{N} \rightarrow S$ be such that

$$
\lim _{T \rightarrow \infty} \frac{1}{T} \sum_{i=1}^{T} \alpha(i)=p .
$$

If $\alpha(i)=s_{v}$ then individual $i$ is of type $s_{v}$. The $v$ th component of the vector $p, p_{v}$, represents the proportion of the population of type $s_{v}$. We assume throughout that the initial population of type $s_{r}, p_{r}$, is positive. 
Let $\Sigma$ be the set of all possible pairwise matchings; i.e.,

$$
\Sigma=\left\{\sigma: \mathbf{N} \rightarrow \mathbf{N} \mid \sigma \text { is bijective and for all } i, \sigma^{2}(i)=i \text { and } \sigma(i) \neq i\right\}^{4}
$$

A few remarks on the conditions that characterize $\Sigma$. The first condition says that each individual is matched exactly once. The second condition says that "if John is matched with Paul then Paul is matched with John." The third condition states that an individual cannot be matched to himself. A random matching scheme is then a probability space $(\Sigma, \mathscr{F}, P)$, where $\mathscr{F}$ is a $\sigma$-algebra of subsets of $\Sigma$ and $P$ is a countably additive probability measure.

We first show that there does not exist a probability space $(\Sigma, \mathscr{F}, P)$ such that for all distinct players $i, j, k \in \mathbf{N}$, the event that player $i$ is matched with player $j$ and the event that player $i$ is matched with player $k$ are equally likely. Suppose that $(\Sigma, \mathscr{F}, P)$ is such a probability space. Denote the probability that player $i$ is matched with player $j$ by

$$
q_{j}=P(\sigma(i)=j)=P(\{\sigma \in \Sigma \mid \sigma(i)=j\}) .
$$

In order for $q_{j}$ to be well defined we need to assume that for all individuals $i, j \in \mathbf{N}$,

$$
\{\sigma \in \Sigma \mid \sigma(i)=j\} \in \mathscr{F} .
$$

Suppose that for $i \neq j, q_{j}=q>0$. Note that since each individual is matched once, the sets $\{\sigma \in \Sigma \mid \sigma(i)=j\}$ and $\{\sigma \in \Sigma \mid \sigma(i)=k\}$ are disjoint. Thus

$$
\begin{aligned}
P(\sigma(i) \in N) & =P\left(\bigcup_{j \in \mathbf{N} \backslash\{i\}} \sigma(i)=j\right) \\
& =\sum_{j \in \mathbf{N} \backslash\{i\}} P(\sigma(i)=j)=\sum_{j \in \mathbf{N} \backslash\{i\}} q=\infty .
\end{aligned}
$$

This clearly contradicts the definition of a probability. Alternatively, if $q=0$ then $P(\sigma(i) \in \mathbf{N})=0$ which is not consistent with the fact that individual $i$ is matched once.

${ }^{4}$ The use of the symbol, $\sigma$, in this paper follows Algebra, where a permutation is denoted by $\sigma$. Gilboa and Matsui [14] use a much bigger matching space when examining the problem of matching two different populations. They define the set of matchings to be $\Omega$ where

$$
\Omega=\{\omega: \mathbf{N} \rightarrow[0,1) \times \mathbf{N} \times \mathbf{N}\} .
$$

The interpretation is as follows. There are a countable number of matches labeled $\omega(1), \omega(2), \ldots$. A match $\omega(k)=(t, i, j)$ consists of individuals $i$ and $j$ being matched at time $t$. 
Clearly the assumption in Eq. (2) and the assumption that all matches are equally likely lead to this contradiction. In order to weaken the set of measurable sets we need to find another way to express the idea that all matches are equally likely. Alternatively, we can relax the assumption that all matches are equally likely.

\section{Summary of Results}

\subsection{A Characterization of Random Matching Schemes}

There are five conditions on the random matching scheme $(\Sigma, \mathscr{F}, P)$ that we consider. Condition I states that for all individual $i, j, k$, the probability with which individual $i$ is matched with individual $j$ equals the probability with which individual $i$ is matched with individual $k$. Condition II states that the probability with which individual $i$ is matched with an individual of type $s_{r}$ equals the proportion of the population of type $s_{r}, p_{r}$. Condition III states that, with probability 1 and for $r \neq v$, the proportion of the population of type $s_{r}$ that is matched with an individual of type $s_{v}$ equals $2 p_{r} p_{v}{ }^{5}$ Condition IV, which is implied by condition III, states that for any $\varepsilon>0$ and for any $\delta>0$, if the population is large enough then with probability greater than $1-\delta$ the proportion of the population of type $s_{r}$ that is matched with an individual of type $s_{v}$ is within $\varepsilon$ of $p_{r} p_{v}$. Condition V states that the random matching scheme does not depend on the individuals' types. Formally,

$$
\begin{aligned}
\text { I. For all } i \in \mathbf{N}, j, k \in \mathbf{N} \backslash i, P[\sigma(i)=j]=P[\sigma(i)=k] \text {. } \\
\text { II. For all } i \in \mathbf{N}, s_{r} \in S, P\left[\alpha(\sigma(i))=s_{r}\right]=p_{r} .
\end{aligned}
$$

III. (SLLN) For all $r \neq v,(1 / n) \sum_{i=1}^{n} \alpha_{r}(i) \alpha_{v}(\sigma(i))$ converges almost surely to $p_{r} p_{v} \cdot{ }^{6}$

IV. (WLLN) For all $r \neq v,(1 / n) \sum_{i=1}^{n} \alpha_{r}(i) \alpha_{v}(\sigma(i))$ converges in probability to $p_{r} p_{v}$.

V. The random matching scheme $(\Sigma, \mathscr{F}, P)$ does not depend on the assignment of types $\alpha$.

We showed in the previous section that condition I cannot be satisfied. Are there matching schemes that satisfy the remaining conditions, or at least a subset of those?

\footnotetext{
${ }^{5}$ The "2" is missing from condition III because the expression is asymmetric in $r$ and $v$.

${ }^{6}$ Note that since the probability is countable additive, if condition I holds, this condition can be replaced by the condition that the random variables $\{\alpha(\sigma(i))\}$ are independent. This is not true if the probability measure is only finitely additive (see [25]).
} 
Proposition 2. There exists a matching scheme for which Condition II and Condition III are satisfied.

This proposition corresponds to Theorem 3 in Section 5 . The random matching scheme works as follows. (i) Each individual rolls an $m$-sided dice where the probability that the outcome is $s_{r}$ is $p_{r}$. (ii) Suppose the $n$th individual is of type $s_{r}$ and rolls an $s_{v}$, where $r \neq v$. Suppose there exists an individual in the population $\{1, \ldots, n-1\}$ who is of type $s_{v}$, has rolled an $s_{r}$, and has yet to be paired. If there are several individuals that satisfy these conditions, pick the individual represented by the lowest integer and match this individual to $n$. (iii) Suppose the $n$th individual is of type $s_{r}$ and rolls an $s_{r}$. Then if there exists another individual of type $s_{r}$, who has rolled an $s_{r}$ and who is not paired yet, match this individual to $n$. Figure 1 gives an example of how the random matching works. Clearly, this matching process satisfies condition II. By the strong law of large numbers the matching rule satisfies condition III.

PROPOSITION 3. There exists a matching scheme for which condition IV and condition $\mathrm{V}$ are satisfied.

This proposition corresponds to Corollary 2 in Section 8 . The random matching scheme described in this theorem works as follows. We put the first 2 individuals in the first urn, the next 4 individuals in the second urn, the next 18 individuals in the third urn, and so on. Specifically, let $k(n)$ be the number of individuals in the $k$ th urn, where $k(1)=2, \phi(n)=\sum_{i=1}^{n} k(i)$, and $k(n)=n \phi(n-1)$. The random matching is generated by drawing individuals, pairwise and without replacement, from each urn. Figure 2 gives an example of how the random matching works.

For large enough urns the law of large numbers will hold. But the urns were formed such that just about all individuals would be placed in a large enough urn. Thus the law of large numbers holds for the set of individuals as a whole.

Proposition 4. There are no matching schemes for which condition II and condition $\mathrm{V}$ hold.

\begin{tabular}{l|c|c|c|c|c|c|c|}
\hline individual & 1 & 2 & 3 & 4 & 5 & 6 & $\ldots$ \\
\hline type & $s_{1}$ & $s_{1}$ & $s_{2}$ & $s_{2}$ & $s_{2}$ & $s_{1}$ & $\ldots$ \\
\hline roll & $s_{1}$ & $s_{2}$ & $s_{1}$ & $s_{2}$ & $s_{2}$ & $s_{1}$ & $\ldots$ \\
\hline match & 6 & 3 & 2 & 5 & 4 & 1 & $\ldots$ \\
\hline
\end{tabular}

FIG. 1. Illustration of the random matching scheme constructed in Proposition 1. 


\begin{tabular}{l|c|c|c|c|c|c|c|}
\hline urn & \multicolumn{2}{|c|}{1} & \multicolumn{4}{c|}{2} & $\ldots$ \\
\hline individual & 1 & 2 & 3 & 4 & 5 & 6 & $\ldots$ \\
\hline match & 2 & 1 & 6 & 5 & 4 & 3 & $\ldots$ \\
\hline
\end{tabular}

FIG. 2. Illustration of the random matching scheme constructed in Proposition 2.

Proof. Suppose condition II holds. Let

$$
\alpha^{n}(i)=\left\{\begin{array}{lll}
s_{2} & \text { if } \quad i \leqslant n \\
\alpha(i) & \text { if } & i>n .
\end{array}\right.
$$

Let

$$
\begin{aligned}
F^{n} & =\left\{\sigma \in \Sigma \mid \alpha^{n}(\sigma(1))=s_{1}\right\} \\
& =\left\{\sigma \in \Sigma \mid \exists i>n \text { such that } \alpha(i)=s_{1} \text { and } \sigma(1)=i\right\} .
\end{aligned}
$$

Clearly, $F^{1} \supset F^{2} \supset \cdots \supset F^{n} \supset \cdots$ and $P\left(\cap_{n} F^{n}\right)=0$. Then, since $\lim _{n \rightarrow \infty} P\left(F^{n}\right)=P\left(\lim _{n \rightarrow \infty} F^{n}\right)$, for $n$ large enough, $P\left(F^{n}\right)<p_{1}$. Contradiction.

Note that the existence of a matching scheme can be thought of as a mechanism design problem. Proposition 4 can then be interpreted as the impossibility of designing a "fair" mechanism if the designer does not know the type of all individuals. Gilboa and Matsui [14] are able to prove the existence of a finitely additive random matching scheme that satisfies condition I. However, countable additivity seems necessary for establishing condition III and condition IV.

\subsection{A Characterization of Repeated Random Matching Schemes for Infinite Populations}

Let $A$ be the set of assignments of types in the population that have a Cesaro average; i.e.,

$$
A=\left\{\alpha \in S^{\mathbf{N}} \mid \lim _{n \rightarrow \infty}(1 / n) \sum_{i=1}^{n} \alpha(i) \text { exists and is strictly positive }\right\} .
$$

Let $\tau: S \times S \rightarrow S$ be the updating rulc; i.c., an individual of type $s_{r}$ who is matched with an individual of type $s_{v}$ becomes a type $\tau\left(s_{r}, s_{v}\right)$. A repeated random matching scheme is described by a probability space $\left(\Sigma^{\infty} \times A^{\infty}, \mathscr{G}\right.$, Prob $)$.

Let $\alpha^{t}(i)$ denote the type of individual $i$ at time $t$. If $\alpha^{t} \in A$, then $p^{t}$ denotes the Cesaro average of $\alpha^{t}$. There are three conditions on the repeated random matching scheme $\left(\Sigma^{\infty} \times A^{\infty}, \mathscr{G}\right.$, Prob $)$ that we consider. Condition A below requires that individual $i$ 's type at time $t, \alpha^{t}(i)$, depends 
on the type of the individual in the previous period, $\alpha^{t-1}(i)$, and the type of the individual that was matched with individual $i$ at time $t-1$, $\alpha^{t-1}\left(\sigma^{t-1}(i)\right)$. Condition B requires that the probability with which individual $i$ is matched with an individual of type $s_{v}$, at time $t$, is $p_{v}^{t}$. Condition $\mathrm{C}$ states that, with probability 1 and for $r \neq v$, the proportion of the population of type $s_{r}$ that is matched with an individual of type $s_{v}$ equals $2 p_{r}^{t} p_{v}^{t}$, for every period $t$. Formally,

A. There exists a measurable set $G \in \mathscr{G}$ such that $\operatorname{Prob}(G)=1$ and for all sequences $\left\{\left(\alpha^{t}, \sigma^{t}\right)\right\} \in G, \alpha^{t}(i)=\tau\left[\alpha^{t-1}(i), \alpha^{t-1}\left(\sigma^{t-1}(i)\right)\right]$.

B. For all $i, s_{r}, t, \alpha^{t} \in A, \operatorname{Prob}\left[\alpha^{t}\left(\sigma^{t}(i)\right)=s_{r} \mid \alpha^{t}\right]=p_{r}^{t}$.

C. There exists a measurable set $G \in \mathscr{G}$ such that $\operatorname{Prob}(G)=1$ and for all $\left\{\left(\alpha^{t}, \sigma^{t}\right)\right\} \in G$, for all $r \neq v$, for all $t, \lim _{n \rightarrow \infty}(1 / n) \sum_{i=1}^{n} \alpha_{r}^{t}(i) \alpha_{v}^{t}\left(\sigma^{t}(i)\right)=$ $p_{r}^{t} p_{v}^{t}$.

The next proposition is a generalization of Proposition 2.

Proposition 5. There exists a dynamical random matching scheme such that conditions $\mathrm{A}, \mathrm{B}$, and $\mathrm{C}$ hold.

This proposition corresponds to Theorem 5 in Section 7. At each period we use the random matching scheme defined in Proposition 1: since there are a countable number of periods it seems clear that Proposition 1 should imply Proposition 4 . The difficulty resides in defining the probability space. ${ }^{7}$ The basic steps in the proof are the following: (i) For each $\alpha \in A$ the matching scheme is defined as in the proof of Proposition 1. (ii) Next we define a matching scheme for all $\alpha \in S^{\mathbf{N}} \backslash A, \quad\left(\Sigma, \mathscr{F}_{\alpha}, P_{\alpha}\right)$, such that $\left(\Sigma, \mathscr{F}_{\alpha}, P_{\alpha}\right)$ is measurable with respect to all $\alpha \in S^{\mathrm{N}}$. The crucial result used to accomplish this is the Kuratowski-Ryll-Nordziewski Theorem. (iii) Then we use the Ionescu Tulcea theorem to define a probability space over $\Sigma^{\infty} \times\left(S^{\mathbf{N}}\right)^{\infty}$. (iv) The rest of the proof consists of restricting the probability space to $\Sigma^{\infty} \times A^{\infty}$.

\subsection{A Characterization of Repeated Random Matching Schemes for Finite Populations}

If the population size is $N$, denote the population by $P(N)=\{1, \ldots, N\}$, and let $\Sigma_{N}$ be the set of all possible matches, where

$$
\begin{aligned}
& \Sigma_{N}=\left\{\sigma: P(N) \rightarrow P(N) \mid \sigma \text { is bijective and for all } i, \sigma^{2}(i)=i\right. \\
&\text { and } \sigma(i) \neq i\} .
\end{aligned}
$$

\footnotetext{
${ }^{7}$ This is similar to the fact that it is much simpler to prove the law of large numbers for coin tosses (just use Chebychev's inequality) than proving that the coin tosses come from a well defined probability space (where we use Kolmogorov's extension theorem).
} 
Let $P_{N}$ be the probability measure over $\Sigma_{N}$ such that all matches are equally likely; i.e.,

$$
M \in 2^{\Sigma_{N}}, P_{N}(M)=\frac{|M|}{\left|\Sigma_{N}\right|} .
$$

Note that the matching scheme $\left(\Sigma_{N}, 2^{\Sigma_{N}}, P_{N}\right)$ satisfies condition 1 and condition V. For any $T>0$, define the $T$-repeated random matching scheme $\left(\Sigma^{T}, 2^{\Sigma^{T}}, P^{T}\right)$, where $2^{\Sigma^{T}}$ is the product $\sigma$-algebra and $P_{N}^{T}$ is the product measure.

Proposition 6. Fix a time period $T$ and consider the stochastic process generated by the law of motion $\tau$ and the random matching scheme $\left(\Sigma^{T}, 2^{\Sigma T}, P^{T}\right)$. Then, for $N$ large enough (where $N$ depends upon $T$ ), the process can be approximated by the deterministic process which is computed by taking the expected outcomes in each period.

This proposition is proved in Section 8.2 .

\section{Construction of a Probability Space Over Matches}

In this section we construct a probability space over the set of matches by considering the events: "the set of matches such that individual $i$ is matched with an individual of type $s_{v}$." Let $S^{\mathrm{N}}$ be "the set of realizations of matching types;" i.e., if $x \in S^{\mathbf{N}}$, then $x(i)$ is the type of $i$ 's match. We first define a probability space over $S^{\mathbf{N}}$ by requiring that the probability with which an individual is matched with an individual of a particular type is equal to the proportion of the population of that type. Then we show that this probability space induces a probability space over matches, $\left(\Sigma, \mathscr{F}_{\alpha}^{2}, P_{\alpha}^{2}\right){ }^{8}$ This probability space is the $\left(P_{\alpha}^{2}\right)$ unique probability space for which the probability with which an individual is matched to an individual of a particular type is equal to the population average. For this probability space each event, "individual $i$ is matched with individual $j$," is not measurable. If we let these events be measurable, as in the probability space $\left(\Sigma, \mathscr{F}_{\alpha}^{1}, P_{\alpha}^{1}\right)$, then these events are not equally likely, although all individuals have the same probability of being matched with an individual of type $s_{v}$ (for all $s_{v} \in S$ ).

We consider each element in $S^{\mathrm{N}}$ as the realization of an infinite sequence of i.i.d. random variables where the probability that $x(i)$ equals $s_{v}$ is $p_{v}$. If $m=6$ and $p_{v}=1 / 6$ (for $v=1, \ldots, 6$ ) we can think of $x$ as the outcome from rolling a dice infinitely many times. Let $\left(S^{\mathrm{N}}, \mathscr{B}, \mu\right)$ be the probability space

\footnotetext{
${ }^{8}$ Thus calling elements in $S^{\mathrm{N}}$ "realizations of matching type" is justified since they can be derived from a random matching scheme.
} 
we just described where $\mathscr{B}$ is the $\sigma$-algebra generated by the finite dimensional rectangles, and $\mu$ is the extension of the probability over the finite dimensional rectangles. ${ }^{9}$ For all types $s_{r}$, let $I_{r}(\alpha) \subset \mathrm{N}$ be the subset of the population of type $s_{r}$; i.e.,

$$
I_{r}(\alpha)=\left\{i \in \mathrm{N} \mid \alpha(i)=s_{r}\right\} .
$$

Note that each set $I_{r}(\alpha)$ is infinite. Let $X_{r v}(\alpha) \subset S^{\mathbf{N}}$ be the set of realizations of the matching process such that the proportion of individuals that are of type $r$ and are matched with individuals of type $v$ is $p_{r} p_{v}$; i.e.,

$$
X_{r v}(\alpha)=\left\{x \in S^{\mathrm{N}} \mid \lim _{T \rightarrow \infty}(1 / T) \sum_{i=1}^{T} \alpha_{r}(i) x_{v}(i)=p_{r} p_{v}\right\}
$$

Then by the strong law of large numbers, $\mu\left(X_{r v}(\alpha)\right)=1 .{ }^{10}$ Let $X_{\alpha}$ be the set of realizations of the matching process such that for all $s_{r}, s_{v} \in S$, the proportion of individuals that are of type $r$ and are matched with individuals of type $v$ is $p_{r} p_{v}$; i.e., $X_{\alpha}=\bigcap_{r} \bigcap_{v} X_{r v}(\alpha)$. Note that since $X_{\alpha}$ is the finite intersection of sets of measure one, $\mu\left(X_{x}\right)=1$.

For all $x \in X_{\alpha}$ and for all types $s_{r}, s_{v}$, let $A_{r v}^{x}$ be the set of players of type $s_{r}$ that are matched with an individual of type $s_{v}$; i.e.,

$$
A_{r v}^{x} \equiv\left\{i \in \mathrm{N} \mid \alpha(i)=s_{r}, x(i)=s_{v}\right\} .
$$

Note that each of the sets $A_{r v}^{x}$ has countably infinite many elements and thus can be enumerated as

$$
A_{r v}^{x}=\left\{a_{r v}^{x}(1), a_{r v}^{x}(2), \ldots\right\},
$$

${ }^{9}$ Formally, a finite dimensional rectangle is a set of the form

$$
B=\left\{x \in X \mid x_{i} \in B_{i} \text { for all } i \text { in } J\right\}
$$

where $J$ is a finite subset of $\mathbf{N}$ and for all $i$ in $J, B_{i}$ is a subset of $S$. Let

$$
\mu_{J}(B)=\prod_{i \in J}\left(\sum_{s \in B_{i}} p_{s}\right) .
$$

Let $\mathscr{B}$ be the $\sigma$-algebra generated by the finite dimensional rectangles. Proposition V.1.2 in [23] shows that there exists a probability over $\left(S^{\mathbf{N}}, \mathscr{B}\right)$ such that for all $J$-dimensional rectangles, $B, \mu(B)=\mu_{J}(B)$.

${ }^{10}$ Let $X_{i}=\alpha_{r}(i) x_{v}(i)$. Then, $\left\{X_{i}\right\}$ is a sequence of independent random variables with finite variance, $\sigma_{i}$. Furthermore, $\sum_{i=1}^{\infty}\left(\sigma_{i} / i^{2}\right)<\infty$, and thus by the Kolmogorov's law of large numbers (see for instance [27, Theorem 6, p. 60]), $\lim _{T \rightarrow x_{i}}(1 / T) \sum_{i=1}^{T} X_{i}=p_{r} p_{r}$ (almost surely). 
where $a_{r v}^{x}(i)<a_{r v}^{x}(i+1)$. For any two different types $s_{r}$ and $s_{v}$ let

$$
\begin{aligned}
& \sigma_{\alpha}^{x}\left(a_{r r}^{x}(i)\right)= \begin{cases}a_{r r}^{x}(i+1) & \text { if } i \text { is odd, } \\
a_{r r}^{x}(i-1) & \text { if } i \text { is even; }\end{cases} \\
& \sigma_{\alpha}^{x}\left(a_{r v}^{x}(i)\right)=a_{v r}^{x}(i), \quad \text { if } \quad r \neq v .
\end{aligned}
$$

Clearly if $x \in X_{x}$, then $\sigma_{\sigma}^{x} \in \Sigma$.

Let $\sigma_{\alpha}: X_{\alpha} \rightarrow \Sigma$ be such that $\sigma_{\alpha}(x)=\sigma_{\alpha}^{\mathrm{r}}$. The function $\sigma_{\alpha}$ is injective since $\alpha \circ \sigma_{x}^{x}=x$.

Next we construct two different probability spaces for matches. In the first probability space the events "individual $i$ is matched with individual $j$ " and "individual $i$ is matched with individual $k$ " are not equally likely. The second probability space is the coarsest for which each subpopulation $I_{r}(\alpha)$ is matched with the population average. Note that for this second probability space the event "individual $i$ is matched with individual $j$ " are not measurable. ${ }^{11}$

Since $\sigma_{\alpha}$ is injective, by identifying $x$ and $\sigma_{\alpha}^{x}$ we can construct a probability over $\Sigma_{\alpha}=\sigma_{\alpha}\left(X_{\alpha}\right)$. Formally, let $\mathscr{F}_{\alpha}$ be the $\sigma$-algebra generated by $\sigma_{\alpha}\left(\mathscr{B} \cap X_{\alpha}\right)$ and let $P_{\alpha}=\mu \circ \sigma_{\alpha}^{-1}$. Then, $\left(\Sigma_{\alpha}, \mathscr{F}_{\alpha}, P_{\alpha}\right)$ is a probability space.

We can extend this probability over all $\Sigma$ by letting $\mathscr{F}_{\alpha}^{1}$ be the $\sigma$-algebra generated by $\mathscr{F}_{x}$ and $\Sigma \backslash \Sigma_{\alpha}$ and letting $P_{\alpha}^{1}(A)=P_{\alpha}\left(A \cap \Sigma_{\alpha}\right)$.

THFORFM $1\left(\sum, \mathscr{F}_{\alpha}^{1}, P_{\alpha}^{1}\right)$ is a probability space for which the event $\{\sigma \in \Sigma \mid \sigma(i)=j\}$ is measurable.

Proof. Let $B_{i}^{n}$ be the event " $i$ is the $n$th player of type $\alpha(i)$ who is matched with an individual of type $\alpha(j)$." Let $N_{i}=\{k \leqslant i \mid \alpha(k)=\alpha(i)\}$. Then

$$
\begin{gathered}
B_{i}^{n}=\bigcup_{\left\{N^{\prime} \subset N_{i}|| N^{\prime} \mid=n\right\}}\left\{x \in S^{\mathrm{N}} \mid x(i)=\alpha(j), \forall k^{\prime} \in N^{\prime}, x\left(k^{\prime}\right)=\alpha(j),\right. \\
\text { and } \left.\forall k^{\prime \prime} \in N_{i} \backslash N^{\prime}, x\left(k^{\prime \prime}\right) \neq \alpha(j)\right\} .
\end{gathered}
$$

Thus $B_{i}^{n}$ is the finite union of rectangles and is thus measurable. Similarly, let $B_{j}^{n}$ be the event " $j$ is the $n$th player of type $\alpha(j)$ who is matched with an individual of type $\alpha(i)$." Clearly, $B_{j}^{n}$ is measurable. Let $B^{n}=B_{i}^{n} \cap B_{j}^{n}$ and let $B=\bigcup_{n \leqslant \min \{i, j\}} B^{n}$. Clearly, $B^{n}$ is measurable and $x \in B$ if and only if $\sigma_{x}(x)(i)=j$. Therefore,

$$
\sigma_{\alpha}(B)=\{\sigma \in \Sigma \mid \sigma(i)=j\} \in \mathscr{F}_{\alpha}^{1},
$$

and thus the event that individual $i$ is matched with individual $j$ is measurable.

\footnotetext{
${ }^{11}$ Recall that in Section 3 we showed that for a probability space over $\Sigma$ either the events "individual $i$ is matched with individual $j$ " are not measurable or they are not equally likely.
} 
The random matching scheme $\left(\Sigma, \mathscr{F}_{\alpha}^{1}, P_{\alpha}^{1}\right)$ has the property that an individual, $i$, is more likely to meet an individual $j$, when this individual is nearby (i.e., $|i-j|$ small), and less likely to meet an individual, $j$, when this individual is far away (i.e., $|i-j|$ big). We define a random matching scheme, $\left(\Sigma, \mathscr{F}_{\alpha}^{2}, P_{\alpha}^{2}\right)$, that does not have this property and such that $P_{\alpha}^{2}$ and $P_{\alpha}^{1}$ agree on $\mathscr{F}_{\alpha}^{2}$. In order to do this, let $\phi_{\alpha}: \Sigma \rightarrow S^{\mathbf{N}}$ be defined by $\phi_{\alpha}(\sigma)=\alpha \circ \sigma$ and let $Y_{\alpha}=\phi_{\alpha}(\Sigma)$. Finally, let $\left(S^{\mathbf{N}}, \mathscr{B}^{\prime}, \mu^{\prime}\right)$ be the completion of the space $\left(S^{\mathbf{N}}, \mathscr{B}, \mu\right)$.

Lemma 1. $X_{\alpha} \subset Y_{\alpha}$. Consequently, $\mu^{\prime}\left(Y_{\alpha}\right)=1$.

Proof. Let $x \in X(\alpha)$. Then $\sigma_{\alpha}(x) \in \Sigma$ and $\phi_{\alpha}\left(\sigma_{\alpha}(x)\right)=x$. Therefore, $x \in Y(\alpha)$. Consequently, $\mu^{\prime}\left(Y_{\alpha}\right)=1$ since $X_{\alpha} \subset Y_{\alpha}$ and $\mu^{\prime}\left(X_{\alpha}\right)=1$.

The probability measure $\mu^{\prime}$ is restricted over $Y_{\alpha}$ by setting

$$
\mathscr{B}_{Y}=\mathscr{B}^{\prime} \cap Y_{\alpha}, \mu_{Y}(A)=\mu^{\prime}\left(A \cap Y_{\alpha}\right) .
$$

Let $\mathscr{F}_{\alpha}^{2}$ be the $\sigma$-algebra on $\Sigma$ generated by $\phi_{\alpha}^{-1}\left(\mathscr{B}_{Y}\right)$ and let $P_{\alpha}^{2}=\mu_{Y} \circ \phi_{\alpha}$.

THEOREM 2. $\left(\Sigma, \mathscr{F}_{\alpha}^{2}, P_{\alpha}^{2}\right)$ is a probability space.

Proof. Follows from the previous lemma, the fact that $\mu\left(X_{\alpha}\right)=1$, and Proposition 2.12 (p. 21) in [3].

For either probability space we have thus proven the following theorem.

THEOREM 3. Let $I_{r}(\alpha)$ be the subset of the population of type $s_{r}$. Suppose that the proportion of the population of type $s_{v}$ is $p_{v}$. Suppose that people are matched at random according to the matching rule $\left(\Sigma, \mathscr{F}_{\alpha}, P_{\alpha}\right)$. Then the proportion of the population $I_{r}(\alpha)$ that is matched with an individual of type $s_{v}$ is $p_{v}$ (almost surely).

\section{Extension of the Probability of THE \\ Realizations of Matches}

In the previous section we defined a probability over the set of realizations of the matching process. In order to do that we had to assume that the assignment of types, $\alpha$, was such that the Cesaro average converges; i.e., $\alpha \in A$ where

$$
A=\left\{\alpha \in S^{\mathrm{N}} \mid \lim _{T \rightarrow \infty} \frac{1}{T} \sum_{i=1}^{T} \alpha(i) \text { exists and is strictly positive }\right\} .
$$


In this section we dispence with this assumption; i.e., we define a probability over the realization of matches for all $\alpha \in S^{\mathbf{N}}$. This is done by means of a measurable extension of the averaging which we denote by $g$.

In order to prove the existence of a measurable extension we need both a topological and a measurable structure for $S^{N}$. The space $S^{N}$ is endowed with the product topology; this makes $S^{\mathbf{N}}$ a complete, separable, metric space ${ }^{12}$ and $\left(S^{\mathbf{N}}, \mathscr{B}\right)$ a measurable space where $\mathscr{B}$ is the Borel $\sigma$-algebra.

Let $G: S^{N} \rightarrow \mathbf{R}^{m}$ be such that for all $\alpha \in S^{N}, G(\alpha)$ is the set of cluster points of $\alpha$; i.e.,

$$
G(\alpha)=\left\{a \in \mathbf{R}^{m} \mid \exists\left\{T_{n}\right\}_{n} \text { such that } \lim _{n \rightarrow \infty} \frac{1}{T_{n}} \sum_{i=1}^{T_{n}} \alpha(i)=a\right\} .
$$

Note that since $\left\{(1 / T) \sum_{i=1}^{T} \alpha(i)\right\}_{T \in \mathrm{N}}$ is an infinite sequence belonging to the $m$-dimensional simplex it has a convergent subsequence and thus $G(\alpha)$ is nonempty.

Lemma 2. The correspondence $G$ is closed-valued and measurable.

Proof. Fix $\alpha \in S^{\mathbf{N}}$, let $\left\{a^{m}\right\}_{m}$ be such that $a^{m} \in G(\alpha)$ and $a^{m} \rightarrow a$. For all $m \in \mathbf{N}$, let $\left\{T_{n}^{m}\right\}_{n}$ be such that

$$
\lim _{n \rightarrow \infty} \frac{1}{T_{n}^{m}} \sum_{i=1}^{T_{n}^{m}} \alpha(i)=a^{m} .
$$

For each $m \in \mathbf{N}$, let $n(m)$ be such that

$$
\left\|\frac{1}{T_{n(m)}^{m}} \sum_{i=1}^{T_{n=1}^{m}(m)} \alpha(i)-a^{m}\right\|<1 / m \quad \text { and } \quad n(m)>n(m-1) \text {. }
$$

Then it is each to check that $\lim _{m \rightarrow \infty}\left(1 / T_{n(m)}^{m}\right) \sum_{i=1}^{T_{n(m)}^{m}} \alpha(i)=a$. Therefore, $a \in G(\alpha)$ and $G(\alpha)$ is closed.

Let $F \subset \mathbf{R}^{m}$ be closed. Then,

$$
\begin{aligned}
G^{-1}(F) & =\left\{\alpha \in S^{\mathbf{N}} \mid G(\alpha) \cap F \neq \varnothing\right\} \\
& =\left\{\alpha \in S^{\mathbf{N}} \mid \exists\left\{T_{n}\right\}_{n} \text { such that } \lim _{n \rightarrow \infty} \frac{1}{T_{n}} \sum_{i=1}^{T_{n}} \alpha(i) \in F\right\} \\
& =\bigcap_{n \in \mathbf{N}} \bigcap_{\tau \in \mathbf{N}} \bigcup_{T>\tau} F_{T, n},
\end{aligned}
$$

${ }^{12}$ One possible metric is $d$, where for all $\alpha, \beta \in S^{\mathrm{N}}$,

$$
d(\alpha, \beta)=\sum_{i \in \mathbb{N}} \frac{|\alpha(i)-\beta(i)|}{2^{i(1+|\alpha(i)-\beta(i)|)} . ~}
$$


where

$$
F_{T, n}=\left\{\alpha \in S^{\mathbf{N}} \mid \frac{1}{T} \sum_{i=1}^{T} \alpha(i) \in B\left(F, \frac{1}{n}\right)\right\}
$$

and

$$
B\left(F, \frac{1}{n}\right)=\left\{a \in \mathbf{R}^{m} \mid \exists b \in F \text { such that }\|a-b\| \leqslant \frac{1}{n}\right\} .
$$

Since for all $T$ and $n, F_{T, n} \in \mathscr{B}, G^{-1}(F) \in \mathscr{B}$ and $G$ is measurable.

LEMma 3. The correspondence $G$ has a measurable selection $g$.

Proof. The Kuratowski-Ryll-Nordziewski Theorem (see for instance Theorem 14.2.1 in [20]) states that any closed-valued $\mathscr{B}$-measurable correspondence into a complete separable metric space has a $\mathscr{B}$-measurable selection. Thus the result follows from Lemma 2.

Note that for all $\alpha \in A, g(\alpha)=\lim _{T \rightarrow \infty}(1 / T) \sum_{i=1}^{T} \alpha(i)$ and thus $g$ is the extension we are looking for.

Let $B$ be a finite measurable rectangle in $S^{\mathrm{N}}$; i.e.,

$$
B=\left\{x \in S^{\mathbf{N}} \mid x(i) \in B_{i} \text { for all } i \text { in } J\right\},
$$

where $J \subset \mathbf{N}$ is a finite set and for all $i$ in $J, B_{i} \subset S$. Then let $\mu(\alpha, B)$ be the probability that if the type of each individual is given by $\alpha$, then for all $i \in J$, individual $i$ is matched with an individual of type $B_{i}$; i.e.,

$$
\mu(\alpha, B)=\prod_{i \in J}\left(\sum_{s \in B_{i}} g_{s}(\alpha)\right) .
$$

The function $\mu(\alpha, \cdot)$ can clearly be extended so that $\left(S^{\mathbb{N}}, \mathscr{B}, \mu(\alpha, \cdot)\right)$ is a probability space.

LEMMA 4. The function $\mu$ is a stochastic kernel.

Proof. In order to check the measurability of $\mu(\cdot, B)$ it is sufficient to consider the case where $B$ is a finite dimensional rectangle [23, p. 75]. For this case, $\mu(\cdot, B)$ is clearly a continuous function of $g(\alpha)$. Therefore, $\mu(\cdot, B)$ is a measurable function.

\section{RePEAted Matching SCheme}

In this section we define the notation to describe a population which is matched infinitely many times, and in which after each period individuals' type may change. We extend the results of Section 5 for this context. Note 
that even if we start with an assignment of types in the population that has a Cesaro average, the updating of individuals' types can be such that the new assignment of types does not have a Cesaro average. In the previous section we showed that we could define a random matching scheme for this case. In this section we show that the random matching scheme is such that in every period the assignment of types has a Cesaro average (almost surely).

Let $\tau: S \times S \rightarrow S$ be such that if at time $t$ an individual of type $s_{v}$ is matched with an individual of type $s_{w}$ then at time $t+1$ the individual is of type $\left.\tau\left(s_{v}, s_{u}\right)\right)^{13}$ In order to guarantee that no type disappears immediately we assume that for all types $s_{r}$ there exist types $s_{v}$ and $s_{w}$ (where $r, v, w$ can be equal) such that $\tau\left(s_{v}, s_{w}\right)=s_{r} \in S$. Let $t: S^{\mathbf{N}} \times S^{\mathbf{N}} \rightarrow S^{\mathbf{N}}$ be such that for all $i \in \mathbf{N}, x, \alpha \in S^{\mathbf{N}}$,

$$
t(x, \alpha)(i) \equiv \tau(\alpha(i), x(i)) .^{14}
$$

If at time $t$ individual $i$ is of type $\alpha(i)$ and is matched with an individual of type $x(i)$ then, at time $t+1$, individual $i$ 's type is $t(\alpha, x)(i)$. For all $x \in S^{\mathbf{N}}$ and for all $\alpha \in S^{\mathbf{N}}$, the functions $t: S^{\mathbf{N}} \times\{x\} \rightarrow S^{\mathbf{N}}$ and $t:\{\alpha\} \times S^{\mathbf{N}} \rightarrow S^{\mathbf{N}}$ are continuous ${ }^{15}$ (and thus measurable) and hence jointly measurable. ${ }^{16}$

${ }^{13}$ Note that this law of motion includes the one considered in Section 2, where

$$
\tau\left(s_{v}, s_{w}\right)=\left\{\begin{array}{lll}
s_{v} & \text { if } & a_{v w} \neq 0 \\
s_{w} & \text { if } & a_{v w}=0 .
\end{array}\right.
$$

The results in this paper show that if the population is countably infinite then Eq. (1) describes the behavior of the process (almost surely).

A more general model would allow for a stochastic law of motion; i.e.,

$$
\tau: S \times S \times[0,1] \rightarrow S,
$$

where $\tau$ is measurable and $(S, \mathscr{P}(S))$ and $([0,1], \mathscr{B}([0,1]))$ are measurable spaces (where $\mathscr{P}(S)$ is the power set and $\mathscr{R}(S)$ is the Borel $\sigma$-algebra). The map becomes stochastic after we define a probability over the measurable space $([0,1], \mathscr{R}([0,1]))$.

${ }^{14}$ If $\tau$ is stochastic then $t: S^{\mathbf{N}} \times S^{\mathbf{N}} \times[0,1]^{N} \rightarrow A$ is such that for all $i \in \mathbf{N}, x, \alpha \in S^{\mathbf{N}}$, $\xi \in[0,1]^{N}$,

$$
t(x, \alpha, \xi)(i) \equiv \tau\left(\alpha(i), x(i), \xi_{i}\right) .
$$

${ }^{15}$ Suppose $\alpha^{n} \rightarrow \alpha$ and let $m>0$. Then there exists an $N$ such that for all $n \geqslant N$ and for all $i \leqslant m, \alpha^{n}(i)=\alpha(i)$. Thus for all $n \geqslant N$ and for all $i \leqslant m, t\left(\alpha^{n}, x\right)(i)=t(\alpha, x)(i)$. Therefore, $t\left(\alpha^{n}, x\right) \rightarrow t(\alpha, x)$ and $t(\cdot, x)$ is continuous. The same proof shows that $t(\alpha, \cdot)$ is continuous.

${ }^{16}$ The proof that the continuity of each section implies joint measurability is in the Appendix. 
Let $Z=S^{\mathbf{N}} \times S^{\mathbf{N}}$ and let $\mathscr{C}=\mathscr{B} \otimes \mathscr{B}$. Let $Q: Z \times \mathscr{C} \rightarrow[0,1]$ be such that for all $(\alpha, x) \in Z$ and all $\left(B, B^{\prime}\right) \in \mathscr{C}$,

$$
Q\left((\alpha, x),\left(B, B^{\prime}\right)\right)=\chi_{B}(t(\alpha, x)) \mu\left(t(\alpha, x), B^{\prime}\right) .{ }^{17}
$$

$Q\left((\alpha, x),\left(\alpha^{\prime}, x^{\prime}\right)\right)$ is the probability that if at time $t$ the population has types assigned by $\alpha$ and is matched according to $x$, then at time $t+1$ the population has types assigned by $\alpha^{\prime}$ and is matched according to $x^{\prime}$.

LEMm 5. The function $Q$ is a transition probability, i.e., for each $C \in \mathscr{C}$, $Q(\cdot, C)$ is measurable and for each $z \in Z, Q(z, \cdot)$ is a probability measure.

Proof. Fix $z \in Z$. Then $Q(z, \cdot)$ is the product of two probability measures and is thus a probability. Fix $C=\left(B, B^{\prime}\right) \in \mathscr{C}$. Note that $Q(\cdot, C)$ is the product of two measurable functions of $t$ and that $t$ is a measurable function of $z$. Therefore, $Q(\cdot, C)$ is a measurable function.

The Ionescu-Tulcea theorem (see for instance [23, Proposition V.1.1]) states that if $\left\{\left(E_{t}, \mathscr{F}_{t}\right)\right\}$ is an infinite sequence of measurable spaces and if $P_{t+1}^{0 \ldots t}$ is a transition probability defined with respect to the spaces

$$
\left(\underset{s=0}{\dot{X}} E_{s}, \underset{s=0}{\otimes} \mathscr{F}_{s}\right) \text { and }\left(E_{t+1}, \mathscr{F}_{t+1}\right)
$$

then there exists a unique probability, $P_{x_{0}}$, on

$$
(\Omega, \mathscr{A})=\mathrm{X}_{t}\left(E_{t}, \mathscr{\mathscr { F } _ { t }}\right)
$$

whose value for every measurable rectangle $\mathrm{X}_{t=1}^{T} F_{t} \mathrm{X}_{s=T+1}^{\infty} E_{s}$ is given by

$$
\begin{aligned}
P_{x_{0}}\left[\underset{t=1}{\mathrm{X}} F_{t}\right]= & 1_{F_{0}}\left(x_{0}\right) \int_{F_{1}} P_{1}^{0}\left(x_{0} ; d x_{1}\right) \int_{F_{2}} P_{2}^{01}\left(x_{0}, x_{1} ; d x_{2}\right) \\
& \cdots \int_{F_{T}} P_{T}^{0 \cdots T-1}\left(x_{0} \cdots x_{T-1} ; d x_{T}\right) .
\end{aligned}
$$

Let $\bar{Z}=\mathrm{X}_{n \in \mathrm{N}} Z$ and $\overline{\mathscr{C}}=\bigotimes_{n \in \mathrm{N}} \mathscr{C}$. Then the Ionescu-Tulcea theorem in conjunction with Lemma 5 gives the following result.

\footnotetext{
${ }^{17}$ For the case where $\tau$ is stochastic let $\lambda$ be the Lebesgue space over $[0,1]^{\mathrm{N}}$. For all $\alpha, x \in S^{\mathbf{N}}$, let $\rho_{\alpha, x}$ be the probability measure on $\left(S^{\mathbf{N}},{ }^{\prime}\right)$ defined by
}

$$
\rho_{\alpha, x}(B)=\lambda\left(\left\{\xi \in[0,1]^{\mathrm{N}} \mid t(\alpha, x, \xi) \in B\right\}\right) .
$$

Then,

$$
Q\left((\alpha, x),\left(B, B^{\prime}\right)\right)=\int_{B} \mu\left(t, B^{\prime}\right) d \rho_{\alpha, x}(t)
$$


THEOREM 4. There is a unique probability $\bar{Q}_{z_{0}}$ over $(\bar{Z}, \overline{\mathscr{C}})$ such that for every finite dimensional rectangle, $C_{1} \times \cdots \times C_{J} \mathrm{X}_{n=J+1}^{\infty} S^{\mathrm{N}}$,

$$
\begin{aligned}
& \bar{Q}_{z_{0}}\left[C_{1} \times \cdots \times C_{J} \underset{n=J+1}{\infty} S^{N}\right] \\
& \quad=\int_{C_{1}} Q\left(z_{0}, z_{1} ; d z_{1}\right) \cdots \int_{C_{j}} Q\left(z_{J-1}, z_{J} ; d z_{J}\right) .
\end{aligned}
$$

Just as in section Section 5, let $X_{x}$ be the set of realizations of the matching rule such that each subpopulation is matched with the population average. By the results in Section 5, for all $\alpha \in A, \mu\left(\alpha, X_{\alpha}\right)=1$.

Lemma 6. The correspondence $X: A \rightarrow S^{\mathrm{N}}$ is measurable and closed valued.

Proof. The proof is exactly the same as the proof of Lemma 2.

Lemma 7. The graph of $X$ is measurable.

Proof. The graph of a closed measurable function is measurable (see [20, Proposition 13.2.2 and Proposition 13.2.4]), thus this result follows directly from Lemma 6 .

THFOREM 5. Suppose that the initial population's types are described by $\alpha_{0} \in A$ and the population is matched according to $x_{0} \in X_{x_{0}}$. Then at every period the assignment of types has a Cesaro average and each subpopulation is matched with the population average (almost surely).

Proof. Let $C=$ graph $X$ and let $z \in C$. Clearly, $\chi_{t(z)}(t(z))=1$ and by the results in Section $4, \mu\left(t(z), X_{t(z)}\right)=1$. Then since $t(z) \in A$ we get that $\left(t(z), X_{t(z)}\right) \in$ graph $X$ and thus,

$$
Q(z, C) \geqslant Q\left(z,\left(t(z), X_{t(z)}\right)\right)=\chi_{t(z)}(t(z)) \mu\left(t(z), X_{t(z)}\right)=1 .^{18}
$$

Since for all $z \in C, Q(z, C)=1$, then for all $J \in \mathbf{N}$,

$$
\begin{aligned}
\bar{Q}_{z_{0}} & {[C \times \cdots \times C \underset{n=J+1}{\mathrm{X}} Z] } \\
& =\int_{C} Q\left(z_{0}, z_{1} ; d z_{1}\right) \cdots \int_{C} Q\left(z_{J-1}, z_{J} ; d z_{J}\right)=1 .
\end{aligned}
$$

\footnotetext{
${ }^{18}$ In order to prove that $Q(z, C)=1$ we had to show that $C$ was measurable. An alternative way of proving the same results is to show that the set $C$ is thick (i.e., $A \in \mathscr{C}$, $A \cap C=\varnothing \Rightarrow P(A)=0)$ and thus $\varnothing(x, C)=1$, where $\varnothing$ is the extension of $Q$ over the trace $\sigma$-algebra $\mathscr{C}(\mathscr{C})$ such that $\widetilde{Q}(A \cap C)=Q(A)$ (see [26, p. 15, Theorem 5]).
} 
Since in each period each subpopulation is matched with the population average, given an initial population, $\alpha$, we can compute the distribution of types at any given period $t, g^{t}(\alpha)$. Specifically, by letting

$$
g^{1}(\alpha)=\sum_{v} \sum_{w} g_{v}(\alpha) g_{w^{*}}(\alpha) \tau\left(s_{v}, s_{w}\right),
$$

and we define recursively $g^{t}(\alpha)$ by

$$
g^{\prime}(\alpha)=g^{t-1}\left(g^{1}(\alpha)\right)
$$

Next we want to show that the probability $\bar{Q}_{z_{0}}$ is generated by some probability space over the set of matches.

Let $\phi: \Sigma^{N} \times A^{N} \rightarrow \bar{Z}$ be such that

$$
\phi\left(\left\{\sigma_{i}\right\},\left\{\alpha_{i}\right\}\right)=\left\{\left(\alpha_{i} \circ \sigma_{i}, \alpha_{i}\right)\right\} .
$$

Let $Y=\phi\left(\Sigma^{N} \times A^{N}\right)$ and let $\left(\bar{Z}, \overline{\mathscr{C}}^{\prime}, Q^{\prime}\right)$ be the completion of the space $\left(\bar{Z}, \overline{\mathscr{C}}^{\prime}, \bar{Q}_{z_{0}}\right)$. Using the same arguments as in Lemma 1 we can show that graph $X \subset Y$ and thus $Q^{\prime}(Y)=1$. The probability $Q^{\prime}$ is restricted over $Y$ by setting

$$
\mathscr{C}_{Y}=\mathscr{C} \cap Y \quad \text { and } \quad Q_{Y}(C)=Q^{\prime}(C \cap Y)
$$

Let $\mathscr{F}$ be the $\sigma$-algebra generated by $\phi^{-1}\left(\mathscr{C}_{Y}\right)$ and let $P=Q_{Y^{\circ}} \phi$. Using the same argument as in Section 4 we get the following result.

THEOREM 6. $\left(\Sigma^{\mathrm{N}} \times A^{\mathrm{N}}, \mathscr{F}, P\right)$ is a probability space.

In many applications we will not be interested in the actual matches but only in the evolution of the population. Let $v: S^{\mathbf{N}} \times \mathscr{B} \rightarrow[0,1]$ be such that for all $\alpha \in S^{\mathbf{N}}$ and $B \in \mathscr{B}$,

$$
v(\alpha, B)=\mu\left(\alpha, t_{\alpha}^{-1}(B)\right) .
$$

For all $\alpha, \alpha^{\prime} \in S^{\mathbf{N}}, v\left(\alpha, \alpha^{\prime}\right)$ is the probability that if at time $t$ the population's types are described by $\alpha$, then at time $t+1$ the population's types are assigned by $\alpha^{\prime}$. Futia [12, Theorem 6.2] proves the following result.

LEMMA 8 . The function $v$ is a transition probability; i.e., for each $B \in \mathscr{B}$, $v(\cdot, B)$ is measurable and for each $\alpha \in S^{\mathbf{N}}, v(\alpha, \cdot)$ is a probability.

Let $\bar{S}=\mathrm{X}_{n \in \mathrm{N}} S^{\mathrm{N}}$ and $\mathscr{\mathscr { B }}=\bigotimes_{n \in \mathrm{N}} \mathscr{B}$. Then, the Ionescu-Tulcea theorem gives the following result. 
THEOREM 7. There is a unique probability $\bar{v}_{\alpha_{0}}$ on $(\bar{S}, \bar{B})$ such that for every finite dimensional rectangle, $B_{1} \times \cdots \times B_{J} \mathbf{X}_{n=J+1}^{\infty} S^{\mathbf{N}}$,

$$
\begin{aligned}
& \bar{v}_{\alpha_{0}}\left[B_{1} \times \cdots \times B_{J} \underset{n=J+1}{\infty} S^{\mathbf{N}}\right] \\
& \quad=\int_{B_{1}} v\left(\alpha_{0}, \alpha_{1} ; d \alpha_{1}\right) \cdots \int_{B_{J}} v\left(\alpha_{J-1}, \alpha_{J} ; d \alpha_{J}\right) .
\end{aligned}
$$

Corollary 1. If $\alpha_{0} \in A$ then $v_{\alpha_{0}}\left[A^{\mathrm{N}}\right]=1$.

Proof. Follows since we showed in Section 4 that $v\left(\alpha_{0}, A\right)=1$.

\section{Matching Schemes That Do Not Depend ON INDIVIDUAL'S TYPES}

Suppose there are finitely many individuals. We represent individuals as balls, where the color of the ball represents the type of the individual. The random matching scheme is given by drawing balls from an urn, two at a time and without replacement. In the first subsection we prove that if the urn is large, the probability with which the pairings behave according to expectation is high. In the second subsection and the third subsection we use this result to characterize a random matching scheme for finite and infinite populations.

\subsection{Some Urn Results}

An urn contains $R$ red balls and $B$ blue balls. $2 b$ balls are drawn in pairs from the urn without replacement (where $2 b \leqslant R+B$ ). Let $I_{j}$ be the random variable which equals 1 if the $j$ th pair drawn is \{red, red $\}$ and equals 0 otherwise. Let $X$ be the random variable that denotes the number of $\{$ red, red $\}$ pairs drawn; i.e.,

$$
X=\sum_{j=1}^{b} I_{j}
$$

Then the expected value of $X, E(X)$, is computed by using the exchangeability of the random variable $I_{j}$.

$$
\begin{aligned}
E(X) & =\sum_{j=1}^{b} P[j \text { th pair is }\{\text { red, red }\}] \\
& =b P[1 \text { st pair is }\{\text { red, red }\}] \\
& =b \frac{R}{R+B} \frac{R-1}{R+B-1}
\end{aligned}
$$


Next we compute the variance of $X, V(X)$.

$$
\begin{aligned}
E(X(X-1))= & E\left(\sum_{i \neq j} I_{i} I_{j}\right) \\
= & \sum_{i \neq j} P[i \text { th pair is }\{\text { red, red }\} \text { and } j \text { th pair is }\{\text { red, red }\}] \\
= & b(b-1) P[1 \text { st pair is }\{\text { red, red }\} \\
& \text { and 2nd pair is }\{\text { red, red }\}] \\
= & b(b-1) \frac{R}{R+B} \frac{R-1}{R+B-1} \frac{R-2}{R+B-2} \frac{R-3}{R+B-3} .
\end{aligned}
$$

(First equality: definition of expectation; second equality: algebra; third equality: exchangeability.) Then,

$$
V(X)=\left[E\left(X^{2}\right)-E(X)\right]+E(X)-[E(X)]^{2} .
$$

Let $N$ be the total number of balls and let $R=p N, B=(1-p) N$. Let $2 b^{N}$ be the number of balls drawn when there are $N$ balls in the urn. Let $X^{N}$ be the random variable which denotes the number of $\{$ red, red $\}$ pairs drawn when there are $N$ balls in the urn and $2 b^{N}$ balls are drawn. Let $N$ be very large so that the terms $(N-1),(N-2), \ldots,(p N-3)$ can be approximated by, respectively, $N, N, \ldots, p N$. Then,

$$
\begin{aligned}
E\left(\frac{1}{b^{N}} X^{N}\right)= & \frac{1}{b^{N}} \frac{b^{N}(p N)^{2}}{N^{2}}=p^{2} ; \\
V\left(\frac{1}{b^{N}} X^{N}\right)= & \frac{1}{\left(b^{N}\right)^{2}}\left[\frac{b^{N}\left(b^{N}-1\right)(p N)^{4}}{N^{4}}\right. \\
& \left.+\frac{b^{N}(p N)^{2}}{N^{2}}-\frac{\left(b^{N}\right)^{2}(p N)^{4}}{N^{4}}\right] \\
= & \frac{1}{b^{N}}\left[\left(b^{N}-1\right) p^{4}+p^{2}-b^{N} p^{4}\right] \\
= & \frac{1}{b^{N}} p^{2}\left(1-p^{2}\right) .
\end{aligned}
$$

By Chebychev's inequality, for all $t>0$,

$$
\begin{aligned}
& P\left(\left|\frac{1}{b^{N}} X^{N}-E\left(\frac{1}{b^{N}} X^{N}\right)\right| \geqslant t\right) \\
& \quad \leqslant \frac{1}{t^{2}} V\left(\frac{1}{b^{N}} X^{N}\right)=\frac{1}{t^{2} b^{N}} p^{2}\left(1-p^{2}\right) .
\end{aligned}
$$


Suppose $b^{N} \rightarrow \infty$ as $N \rightarrow \infty$. Then, $\left(1 / b^{N}\right) X^{N}$ converges in probability to $p^{2}$. Similarly the proportion of $\{$ blue, blue $\}$ pairs converges in probability to $(1-p)^{2}$ and the proportion of \{blue, red \} pairs converges in probability to $2 p(1-p)$. Suppose an urn contains balls of type $s_{1}, \ldots, s_{m}$ in proportion $p_{1}, \ldots, p_{m}$. If we label the balls of type $s_{r}$ "red," by the above result we get that the frequence of $\left\{s_{r}, s_{r}\right\}$ pairs approaches $p_{r}^{2}$. If we label the balls of type $s_{r}$ and $s_{v}$ "red," the frequency of pairs $\left\{s_{r}, s_{v}\right\},\left\{s_{r}, s_{r}\right\},\left\{s_{v}, s_{v}\right\}$ approaches $\left(p_{v}+p_{r}\right)^{2}$. Since we know that the frequency of $\left\{s_{r}, s_{r}\right\}$ and $\left\{s_{v}, s_{v}\right\}$ pairs approaches $p_{r}^{2}+p_{v}^{2}$, then the frequency of pairs $\left\{s_{r}, s_{v}\right\}$ must approach $2 p_{r} p_{v}$.

Proposition 7. As the size of the urn goes to infinity the proportion of $\left(s_{r}, s_{v}\right)$ pairs converges in probability to $2 p_{r} p_{v}$.

\subsection{A Matching Scheme for Finite Populations and Finite Number of Periods}

A justification of the analysis of the deterministic model for a finite population is that the modeler is just interested in examining the law of motion for a finite number of periods, $T$, and that for a large enough population the deterministic model is a good approximation of the stochastic model. This section proves this conjecture.

Let $p_{v}^{t}$ be the expected proportion of individuals of type $s_{v}$ at time $t$. The initial proportion $p^{0}$ is given while the other proportions are computed by assuming that each type is matched with the population average. If the population size is $N$, the population is denoted by $P(N)$, where $P(N)=\{1, \ldots, N\}$. For any period $t=1, \ldots, T$, let $\alpha^{t}: P(N) \rightarrow S$ denote the assignment of types in the population. The initial population $\alpha^{0}$ is given while the populations in the other periods are obtained by the law of motion $\tau$ (which is described in Section 7) and the matching rule. The matching scheme is the following: individuals are represented by balls in an urn which are drawn pairwise without replacement. Individuals drawn simultaneously are matched to one another. As usual a match is represented by $\sigma$.

THEOREM 8. Consider the random matching scheme we just defined. Then for any $T>0, \varepsilon>0$ and for any $\delta>0$ there exists a positive integer $N^{\prime}$ such that for all population sizes $N>N^{\prime}$ and all types $s_{v}$ the following holds: with probability greater than $1-\delta$, the proportion of the population $P(N)$ of type $s_{v}$ at time $T,(1 / N) \sum_{i=1}^{N} \alpha_{v}^{T}(i)$, is within $\varepsilon$ of $p_{v}^{T}$.

Proof. The idea behind the proof is the following. We find a lower bound for the proportion of the population which is matched according to the population average in every period (with probability $1-v$ ). The lower 
bound and $1-v$ can be made arbitrarily close to 1 by taking the population to be large enough. This allows us to find a subpopulation which behaves exactly according to the deterministic model. Again the proportion of the population in this subpopulation can be made arbitrarily close to one by taking the population to be large enough.

Fix $T>0, \varepsilon>0$, and $\delta>0$. Let $\xi>0$ be such that $(1-(\varepsilon / \xi))^{T}>1-\varepsilon$, let $v$ be such that $(1-v)^{T}>1-\delta$, let $p=\min _{v, r \leqslant T} p_{v}^{t}$, and let $N$ be greater than $N^{\prime}$ where

$$
N^{\prime}=\frac{T \xi^{2} m(m-1)}{2 \delta \varepsilon^{2} \underline{p}^{4}}\left(1-\frac{\varepsilon}{\xi}\right)^{T-1}
$$

From the results in the previous subsection we know that

$$
P\left(\left|p_{r}^{0} p_{v}^{0}-\frac{1}{N} \sum_{i \in P(N)} \alpha_{r}(i) \alpha_{v}(\sigma(i))\right| \geqslant \frac{t}{2}\right) \leqslant \frac{2}{t^{2} N} .
$$

Let $t=(2 \varepsilon / \xi) p^{2}$ and replace $N$ by its lower bound (for the moment we do not bother with the term $(1-(\varepsilon / \xi))^{T-1}$ in $\left.N\right)$. Then

$$
P\left(\left|p_{r}^{0} p_{v}^{0}-\frac{1}{N} \sum_{i \in P(N)} \alpha_{r}(i) \alpha_{v}(\sigma(i))\right| \geqslant \frac{\varepsilon}{\xi} \underline{p}^{2}\right) \leqslant \frac{v}{m(m-1)} .
$$

Consequently,

$$
P\left(\max _{r, v}\left|p_{r}^{0} p_{v}^{0}-\frac{1}{N} \sum_{i \in P(N)} \alpha_{r}(i) \alpha_{v}(\sigma(i))\right| \geqslant \frac{\varepsilon}{\xi} \underline{p}^{2}\right) \leqslant v .
$$

Some more algebraic manipulations give

$$
P\left(\max _{r, v} \frac{\left|p_{r}^{0} p_{v}^{0}-(1 / N) \sum_{i \in P(N)} \alpha_{r}(i) \alpha_{v}(\sigma(i))\right|}{p_{r} p_{v}}<\frac{\varepsilon}{\xi}\right) \geqslant 1-v .
$$

Thus there exists a subpopulation, $P_{1}$, such that for all $r$ and $v$,

$$
p_{r}^{0} p_{v}^{0}=\sum_{i \in P_{1}} \alpha_{r}(i) \alpha_{v}(\sigma(i))
$$

and $\left|P_{1}\right| \geqslant(1-(\varepsilon / \xi)) N$. Since subpopulation $P_{1}$ is matched according to expectations, after the updating of types, the distributions of individuals in population $P_{1}$ is given by $p^{1}$. Note also that since $N^{\prime}$ has a term $(1-(\varepsilon / \xi))$ which we did not use in computing bounds, population $P_{1}$ is large enough to get exactly the same bounds we computed above. Thus, with probability greater than $1-v$, there is a subpopulation $P_{2}$ of population $P_{1}$ such that 
individuals are matched according to the population average, and $\left|P_{2}\right|>(1-(\varepsilon / \xi))\left|P_{1}\right|>(1-(\varepsilon / \xi))^{2} N$.

Consequently, with probability greater than $(1-v)^{T}>1-\delta$, the proportion of individuals matched according to the correct proportions is $(1-(\varepsilon / \xi))^{T}>1-\varepsilon$, which gives the desired result.

\subsection{A Matching Scheme for Infinite Populations}

Let $k, \phi: \mathbf{N} \rightarrow \mathbf{N}$ be defined recursively by $k(1)=2, \phi(n)=\sum_{i=1}^{n} k(i)$, and $k(n)=n \phi(n-1)$. For all $i \in \mathbf{N}$, let

$$
\begin{aligned}
U_{n}= & \{\phi(n-1), \phi(n-1)+1, \ldots, \phi(n)\} ; \\
D_{n}= & \left\{d: U_{n} \rightarrow U_{n} \mid d \text { is bijective and for all } i \in \mathbf{N},\right. \\
& \left.d(i) \neq i \text { and } d^{2}(i)=i\right\} .
\end{aligned}
$$

(Here $U$ stands for "urn," $D$ stands for "draws," $k(n)$ is the number of individuals in urn $n, \phi(n)$ is the number of individuals in all the urns up to urn $n$.) Let $\mathscr{D}_{n}=2^{D_{n}}$ and for all $D \in \mathscr{D}_{n}$, let

$$
P_{n}(A)=\frac{|A|}{\left|D_{n}\right|} .
$$

Clearly, $\left(D_{i}, \mathscr{D}_{i}, P_{i}\right)$ is a probability space. Let $D=\mathrm{X}_{i=i}^{\infty} D_{i}$, let $\mathscr{D}=\bigotimes_{i=1}^{\infty} \mathscr{D}_{i}$, and let $P=\prod_{i=1}^{\infty} P_{i}$. Then by the definition of product probability, $(D, \mathscr{D}, P)$ is a probability space. Let $d \in D$; then $d(i)=j$ stands for "individual $i$ is matched with individual $j$." $P(d)$ denotes the probability with which the match $d$ occurs.

Let $S=\left\{s_{1}, \ldots, s_{m}\right\}$ where we identify $s_{r}$ with the $m$ dimensional vector with 1 on the $r$ th component and 0 on the other components. Let $\alpha: \mathbf{N} \rightarrow S$ be such that $\lim _{N \rightarrow \infty}(1 / N) \sum_{i=1}^{N} \alpha(i)=p$. Note that in the limit, the average type in an urn is the same as in the population at large; i.e.,

$$
\lim _{n \rightarrow \infty} \frac{1}{k(n)} \sum_{i \in U(n)} \alpha(i)=p
$$

By the result in Section 8.1 (where $b^{N} \equiv N$ ),

$$
(\forall k>0) \lim _{n \rightarrow \infty} P\left[\left|\frac{1}{k(n)} \sum_{i \in U(n)} \alpha_{r}(i) d_{r}(\alpha(i))-p_{r}^{2}\right|>k\right]=0 .
$$

Let $\phi^{-1}(n)=\max \{i \in \mathbf{N} \mid \phi(i) \leqslant n\}$. Note that by the construction of $U(n)$ and the results in the previous section that 


$$
\begin{aligned}
\lim _{n \rightarrow \infty} E & {\left[\frac{1}{n} \sum_{i=1}^{n} \alpha_{r}(i) d_{r}(\alpha(i))\right] } \\
= & \lim _{n \rightarrow \infty} E\left[\frac{1}{n} \sum_{i \in U\left(\phi^{-1}(n)\right)} \alpha_{r}(i) d_{r}(\alpha(i))\right. \\
& \left.+\frac{1}{n} \sum_{i=\phi\left(\phi^{-1}(n)\right)+1}^{n} \alpha_{r}(i) d_{r}(\alpha(i))\right] \\
= & \lim _{n \rightarrow \infty} \frac{k\left(\phi^{-1}(n)\right)}{n} p_{r}^{2}+\frac{n-\phi\left(\phi^{-1}(n)\right)-1}{n} p_{r}^{2} \\
= & p_{r}^{2} .
\end{aligned}
$$

Furthermore, since the draws in one urn are independent from the draws in other urns,

$$
\begin{aligned}
V\left[\frac{1}{n} \sum_{i=1}^{n} \alpha_{r}(i) d_{r}(\alpha(i))\right] \\
=\lim _{n \rightarrow \infty} V\left[\frac{1}{n} \sum_{i \in U\left(\phi^{-1}(n)\right)} \alpha_{r}(i) d_{r}(\alpha(i))\right. \\
\left.\quad+\frac{1}{n} \sum_{i=\phi\left(\phi^{-1}(n)\right)+1}^{n} \alpha_{r}(i) d_{r}(\alpha(i))\right] \\
=\lim _{n \rightarrow \infty} \frac{1}{k\left(\phi^{-1}(n)\right)}\left[\frac{k\left(\phi^{-1}(n)\right)}{n}\right]^{2} p_{r}^{2}\left(1-p_{r}^{2}\right) \\
\quad+\frac{1}{n-\phi\left(\phi^{-1}(n)\right)-1}\left[\frac{n-\phi\left(\phi^{-1}(n)\right)-1}{n}\right]^{2} p_{r}^{2}\left(1-p_{r}^{2}\right) \\
=0 .
\end{aligned}
$$

Thus by Chebychev's inequality we get the following theorem.

THEOREM 9. $(1 / n) \sum_{i=1}^{n} \alpha_{r}(i) d_{r}(\alpha(i))$ converges in probability to $p_{r}^{2}$.

By the same reasoning than the one in the last paragraph of Section 9.1 we get the following corollary.

CoROllary 2. $(1 / n) \sum_{i=1}^{n} \alpha_{r}(i) d_{v}(\alpha(i))$ converges in probability to $p_{r} p_{v}$.

\section{Conclusion}

Biologists and economists have analyzed populations where each individual interacts with randomly selected individuals. The random 
matching generates a very complicated stochastic system. Consequently biologists and economists have approximated such a system with a deterministic system. The justification for such an approximation is that the population is assumed to be very large and thus some law of large numbers must hold. In the paper we give an example for which this assumption does not hold. This does not mean that this kind of approximation may never hold, but that the correctness of the approximation depends on properties of the law of motion.

This paper gives a characterization of random matching schemes for countably infinite populations. In particular this paper shows that there exists a random matching scheme such that the stochastic system and the deterministic system are the same. Economists and biologists have assumed that the probability with which an individual is matched with a particular subpopulation equals the proportion of the population in that subpopulation. This paper shows that, for a countable population, this is possible only if the random matching scheme depends on the assignment of types in the population.

The problem described in the previous paragraph arises only if we are interested in infinite populations. If we examine the behavior of the process for finitely many periods, we conclude that if the population is large enough, then there is a matching scheme such that the deterministic process provides a good approximation of the stochastic process. In proving this result, we provide an upper bound on deviation (Eq. (3)) which should be useful in a variety of applications (in simulations of neural networks, for instance). However, if we consider the case of finite populations and an infinite number of periods we may run into problems as was shown in Section 2.

\section{APPENDIX}

The following lemmas prove Proposition 1. Let $\Lambda=\left\{p \in \mathbf{R}_{+}^{3} \mid p_{1}+p_{2}+\right.$ $\left.p_{3}=1\right\}, \partial \Delta=\left\{p \in \Delta \mid p_{1} p_{2} p_{3}=0\right\}, v_{1}=(1,0,0), v_{2}=(0,1,0), v_{3}=(0,0,1)$. Let $p_{0} \in \Delta \backslash \partial \Delta$ be the initial population, let $p^{t}=R^{t} p^{0}$, and let $\omega$ be the set of accumulation points of the orbit $\left\{p^{t}\right\}$.

\section{LEMMA 9. The set of accumulation points is a subset of the boundary.}

Proof. Suppose $p$ is an accumulation point which is not on the boundary of the simplex; i.e., $p \in \omega \backslash \partial \Delta$. Weising [32] proves that the function $W(p)=\left(1 / p_{1} p_{2} p_{3}\right)$ is strictly increasing along any trajectory. Thus all convergent subsequences must converge to a point on the set $L \subset W^{-1}(W(p))$. Note that by the properties of limit sets $R(L)=L$ but that for each 
$y \in R(L)$ and $z \in L$, we have $W(y)>W(z)$, which is clearly a contradiction.

LEMMA 10. The set of accumulation points includes the three vertices.

Proof. Since $\Delta$ is compact, the set of accumulation points, $\omega$, is nonempty.

We first show that the set of accumulation points includes at least one vertex. Suppose $p$ is a limit point (thus a point on a boundary) which is not a vertex and let the subsequence $\left\{t_{n}\right\}$ be such that $\lim _{n \rightarrow \infty} p^{t_{n}}=p$. Without loss of generality suppose that $p \in\left\{y \in \Delta \mid y_{3}=0\right\}$. Note that $\lim _{n, \infty} R^{n}(p)=v_{2}$. Thus the subsequence $\left\{p^{t_{n}+n}\right\}$ is such that $\lim _{n \rightarrow \infty} p^{t_{n}+n}=v_{2}$.

Note that if $p$ is such that $p_{1}=0$ and $p_{2} p_{3}>0$, then for any $\varepsilon>0$ there is a large enough $N$ such that $\left|p^{N}-v_{3}\right|<\varepsilon$. Since $R^{n}$ is continuous, if $p^{t}$ is close enough to $p$ then $\left|p^{t+N}-v_{3}\right|<\varepsilon$. Consequently $v_{3}$ is an accumulation point. Similarly we can show that $v_{1} \in \omega$.

LEMMA 11. The set of accumulation points is infinite.

Proof. Let $B\left(v_{i}, \varepsilon\right)$ be the open ball of radius $\varepsilon$ around $v_{i}$; i.e., $B\left(v_{i}, \varepsilon\right)=$ $\left\{p \in \Delta|| p-v_{i} \mid<\varepsilon\right\}$. Since $R$ is continuous and $R\left(v_{i}\right)=v_{i}$, there exists a scalar $\varepsilon>0$ such that if $i \neq j$ then $R\left(B\left(v_{i}, \varepsilon\right)\right) \cap B\left(v_{j}, \varepsilon\right)=\varnothing$. Since the vertices are accumulation points of the trajectory $\left\{p^{t}\right\}$, then $p^{t} \in B\left(v_{i}, \varepsilon\right)$ for infinitely many integers $t$. Thus for infinitely many $t, p^{t} \in I \equiv A \backslash \bigcup_{i} B\left(v_{i}, \varepsilon\right)$. Since the set $I$ is compact, there exists a subsequence converging to $p \in I$. Thus $p \in \omega \backslash \bigcup_{i} v_{i}$. Then for all $t, R^{t} p \in \omega$ and for all $t \neq s, R^{\prime} p \neq R^{s} p$ (since $W$ is strictly increasing on $R^{\prime} p$ ).

The following theorem is used in Section 7.

THEOREM 10. Let $(X, \mathscr{B})$ be a measure space where $X$ is a separable complete metric and $\mathscr{B}$ is the Borel $\sigma$-algebra. Let $f: X \times X \rightarrow X$ be such that for all $x \in X, f:\{x\} \times X \rightarrow X$ and $f: X \times\{x\} \rightarrow X$ are continuous. Then $f$ is measurable.

Proof. Since $X$ is a separable metric space, the Borel $\sigma$-algebra $\mathscr{B}$ has a denumerable subfamily, $\mathscr{D}$ generating $\mathscr{B}$ (see for instance [24, Theorem 1.8]). Let

$$
\mathscr{D}=\left\{D_{1}, \ldots, D_{n}, \ldots,\right\}
$$

Let

$$
\begin{aligned}
\mathscr{F}^{n} & =\left\{F_{1} \cap F_{2} \cap \cdots \cap F_{n} \mid \text { where } F_{i}=D_{i} \text { or } F_{i}=X \backslash D_{i}\right\} \\
& \equiv\left\{F_{1}^{n}, \ldots, F_{m(n)}^{n}\right\} .
\end{aligned}
$$


Note that $\mathscr{F}_{n}$ is a partition of $X$ and that $\mathscr{\mathscr { F }}_{n} \subset \mathscr{R}$. For all $i$ and $n$ choose $y_{i}^{n}$ such that $y_{i}^{n} \in F_{i}^{n}$. Finally, let

$$
f_{n}(x, y)=\sum_{i=1}^{m(n)} f\left(x, y_{i}^{n}\right) \chi_{X \times F_{i}^{n}} .
$$

Note that the continuity of $f: X \times\{c\} \rightarrow X$ easily implies the continuity of the function $g: X \times X \rightarrow X$, where $g(x, y)=f(x, c)$. Thus $f_{n}(x, y)$ is a measurable function. Fix $y \in X$ and for all $n$ let $i(n)$ be such that $y \in F_{i(n)}^{n}$. Note that $F_{i(n)}^{n} \subset F_{i(n-1)}^{n-1}$ and that $F_{i(n)}^{n} \downarrow\{y\}$. Then,

$$
\lim _{n \rightarrow \infty} f_{n}(x, y)=\lim _{n \rightarrow \infty} f\left(x, y_{i(n)}^{n}\right)=f\left(x, \lim _{n \rightarrow \infty} y_{i(n)}^{n}\right)=f(x, y) \text {. }
$$

The function $f$ is hence the pointwise limit of a sequence of measurable functions and is thus measurable.

\section{REFERENCES}

1. K. Binmore and L. Samuelson, Evolutionary stability in repeated games played by finite automata, mimeograph, 1990.

2. R. Boylan, "Evolutionary Equilibria Resistant to Mutation," Social Science Working paper No. 729, California Institute of Technology, 1990.

3. L. Breiman, "Probability," Addison-Wesley, Reading, MA., 1968.

4. L. L. Cavalli-Sforza and W. F. Bodmer, "The Genetics of Human Populations," Freeman, San Francisco, 1971.

5. V. Crawford, "Lcarning and Mixcd-Strategy Equilibria in Evolutionary Games," Discussion paper 88-53, University of California, San Diego, 1988.

6. V. CRAwFord, “An 'Evolutionary' Explanation of Van Huyck, Battalio, and Beil's Experimental Results on Coordination," Discussion paper 89-28, University of California, San Diego, 1989.

7. M. Feldman and C. Gilles, An expository note on individual risk without aggregate uncertainty, J. Econ. Theory 35 (1985), 26-32.

8. W. Feller, "An Introduction to Probability Theory and its Applications," 3rd cd., Vol. I, Wiley, New York, 1967.

9. D. Friedman, Evolutionary games in economics, Econometrica 59 (1991), 637-666.

10. D. Fudenberg and D. Levine, Steady state learning and self-confirming equilibrium, mimeograph, University of California, Los Angeles, 1990.

11. D. Fudenberg AND E. Maskin, Evolution and cooperation in noisy repeated games, Amer. Econ. Rev. 80 (1990), 274-279.

12. C. A. FUtiA, Invariant distributions and the limiting behavior of Markovian economic models, Econometrica 50 (1982), 377-408.

13. D. GALE, Bargaining and competition part I: Characterization, Econometrica 54 (1986).

14. I. Gilboa and A. Matsui, "A Model of Random Matching," Discussion paper No. 887, Northwestern University, 1990.

15. E. Green, "Individual-Level Randomness in a Nonatomic Population," Working paper 227, University of Pittsburgh, 1989. 
16. G. H. HaRDY, Mendelian proportions in mixed population, Science 28 (1908), 49-50. Reprinted in "Classic Papers in Genetics," (J. H. Peters, Ed.) Englewood Cliffs, NJ: Prentice-Hall, 1959.

17. J. Hofbauer and K. Sigmund, "The Theory of Evolution and Dynamical Systems," Cambridge Univ. Press, Cambridge, 1988.

18. K. JUDD, The law of large numbers with a continuum of iid random variables, J. Econ. Theory 35 (1985), 19-25.

19. G. KallianPUR, "Stochastic Filtering Theory," Springer-Verlag, New York, 1980.

20. E. Klein ANd A. Thompson, "Theory of Correspondences," Wiley, New York, 1984.

21. V. Losert AND E. Akin, Dynamics of games and genes: Discrete versus continuous time, J. Math. Biol. 17 (1983), 241-251.

22. J. NACHBAR, "Evolutionary" selection dynamics in games: Convergence and limit properties, Int. J. Game Theory 19 (1990), 59-89.

23. J. Neveu, "Mathematical Foundations of the Caiculus of Probability," Holden-Day, San Francisco, 1965.

24. K. R. Parthasarathy, "Probability Measures on Metric Spaces," Academic Press, New York, 1967.

25. S. Ramakrishnan and W. D. SUdDerTh, A sequence of coin toss variables for which the strong law fails, Amer. Math. Mon. 95 (1988), 939-941.

26. M. M. RAO, "Foundations of Stochastic Analysis," Academic Press, New York, 1981.

27. M. M. Rao, "Probabilily Theory with Applications," Academic Press, New York, 1984.

28. L. SAMUELSON, Evolutionary foundations of solution concepts for finite, two player, normal-form games, in "Theoretical Aspects of Reasoning about Knowledge" (M. Y. Vardi, Ed.), pp. 211-225, Morgan Kaufmann, Los Altos, 1988.

29. H. UHLIG, "A Law of Large Numbers for Large Economies," Working paper 342, Federal Reserve Bank of Minneapolis, 1988.

30. E. van Damme, "Stability and Perfection of Nash Equilibria," Springer-Verlag, Berlin, 1987.

31. W. Weinkerg, On the demonstration of heredity in man, reprinted in "Papers on Human Genetics," (S. H. Boyer, Ed.) Englewood Cliffs, N.J.: Prentice-Hall, 1963, 1908.

32. F. Weising, "Evolutionary Stability and Dynamics Stability in Generalized 'RockScissors-Paper' Games," Working paper No. 27, University of Bielfeld, 1989. 